DOI:

10.1038/nri2185

\title{
A new way of sticking the ends together
}

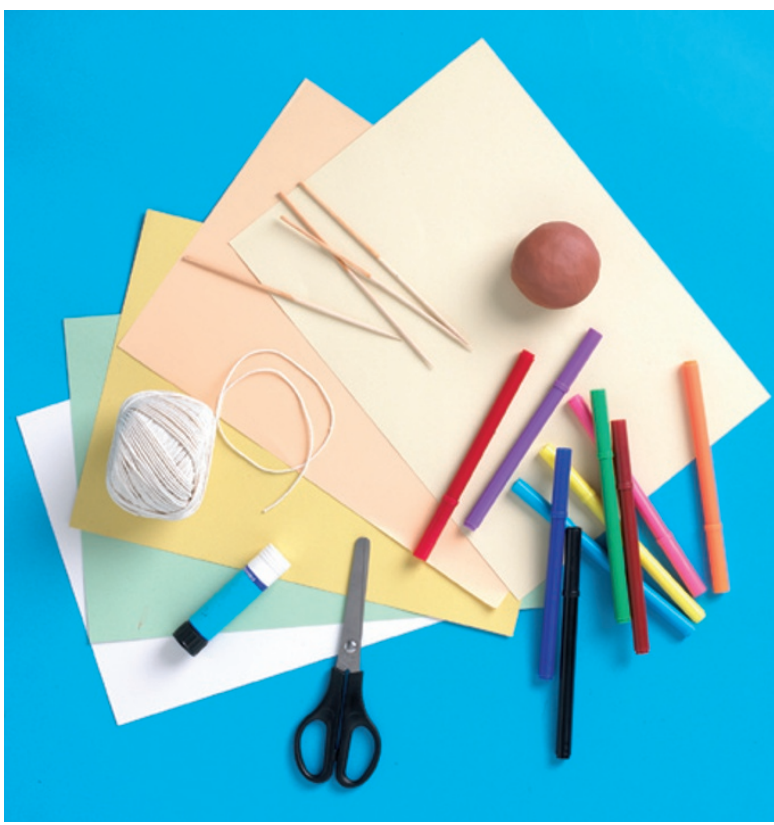

To generate different subtypes of immunoglobulin, B cells must first undergo $\mathrm{V}(\mathrm{D}) \mathrm{J}$ recombination during development, followed by classswitch recombination (CSR) after cell maturation. These processes involve the rearrangement of immunoglobulin variable- and constant-region exons, respectively. For this to occur, double-strand breaks (DSBs) must be introduced into the DNA, which are then repaired by means of end joining. The 'classical' non-homologous end-joining (C-NHEJ) pathway is essential for the repair of DSBs during $\mathrm{V}(\mathrm{D}) \mathrm{J}$ recombination, and it has been suggested that CSR also uses the C-NHEJ pathway. Alt and colleagues, however, have now identified an alternative DNA-repair pathway that is used during CSR.

During V(D)J recombination the C-NHEJ pathway joins non-homologous ends to form 'direct' joins but can also join ends with microhomologies. An 'alternative' end-joining pathway has been described that is biased towards microhomology joins and, in the absence of factors associated with C-NHEJ, has been implicated in oncogenic translocations. However, as this alternative pathway does not rescue $\mathrm{V}(\mathrm{D}) \mathrm{J}$ recombination, it was thought not to function efficiently on DSBs.

Here, the authors generated mutant mice with mature B cells that were deficient for either of the two most specific C-NHEJ factors, XCCR4 or DNA ligase 4 (LIG4), and determined the levels of CSR in activated B cells. They found, using three different detection methods, that although the levels of switched immunoglobulin were reduced in B cells deficient for either XCCR4 or LIG4 compared with control B cells, substantial levels of class switching (25-50\%) still occurred in these cells compared with cells from mice deficient in activation-induced cytidine deaminase (AID; an enzyme required for CSR). This indicates that although there are reduced levels of class switching in the absence of the C-NHEJ pathway, end joining still occurs at a robust level.

To determine the nature of this alternative DNA repair pathway, the authors compared CSR junctions in B cells from Xccr4-deficient and control mice. In control cells $30-60 \%$ of the CSR junctions were direct joins and the rest were short microhomology joins. By contrast, no direct joins were detected in CSR junctions in Xccr4-deficient B cells and most of the CSR junctions were longer microhomology joins, with the rest containing insertions. Interestingly, in the absence of C-NHEJ, a high proportion of breaks (about 20\%) in the immunoglobulin heavy-chain locus were joined to the ends of other chromosomes thereby generating translocations, which indicates that this pathway might be inherently 'error prone.'

So, this study shows that an alternative pathway can be used in B cells to join DSBs during CSR. This robust pathway is fundamentally different from the C-NHEJ pathway in that it occurs in the absence of XCCR 4 and LIG4, it does not result in direct joins and it prefers microhomology joins.

Olive Leavy

ORIGINAL RESEARCH PAPER Yan, C. T. et al.

$\mathrm{IgH}$ class switching and translocations use a

robust non-classical end-joining pathway. Nature 22 August 2007 (doi:10.1038/nature06020) 